\title{
Mathematical Analysis of a Generalized Chiral Quark Soliton Model
}

\author{
Asao ARAI \\ Department of Mathematics, Hokkaido University, Sapporo, 060-0810, Japan \\ E-mail: arai@math.sci.hokudai.ac.jp \\ Received October 18, 2005, in final form January 25, 2006; Published online February 03, 2006 \\ Original article is available at http://www.emis.de/journals/SIGMA/2006/Paper018/
}

\begin{abstract}
A generalized version of the so-called chiral quark soliton model (CQSM) in nuclear physics is introduced. The Hamiltonian of the generalized CQSM is given by a Dirac type operator with a mass term being an operator-valued function. Some mathematically rigorous results on the model are reported. The subjects included are: (i) supersymmetric structure; (ii) spectral properties; (iii) symmetry reduction; (iv) a unitarily equivalent model.
\end{abstract}

Key words: chiral quark soliton model; Dirac operator; supersymmetry; ground state; symmetry reduction

2000 Mathematics Subject Classification: 81Q10; 81Q05; 81Q60; 47N50

\section{Introduction}

The chiral quark soliton model (CQSM) [5] is a model describing a low-energy effective theory of the quantum chromodynamics, which was developed in 1980's (for physical aspects of the model, see, e.g., [5] and references therein). The Hamiltonian of the CQSM is given by a Dirac type operator with iso-spin, which differs from the usual Dirac type operator in that the mass term is a matrix-valued function with an effect of an interaction between quarks and the pion field. It is an interesting object from the purely operator-theoretical point of view too. But there are few mathematically rigorous analyses for such Dirac type operators (e.g., [2], where the problem on essential self-adjointness of a Dirac operator with a variable mass term given by a scalar function is discussed).

In the previous paper [1] we studied some fundamental aspects of the CQSM in a mathematically rigorous way. In this paper we present a slightly general form of the CQSM, which we call a generalized CQSM, and report that results similar to those in [1] hold on this model too, at least, as far as some general aspects are concerned.

\section{A Generalized CQSM}

The Hilbert space of a Dirac particle with mass $M>0$ and iso-spin $1 / 2$ is taken to be $L^{2}\left(\mathbb{R}^{3} ; \mathbb{C}^{4}\right) \otimes \mathbb{C}^{2}$. For a generalization, we replace the iso-spin space $\mathbb{C}^{2}$ by an abitrary complex Hilbert space $\mathcal{K}$. Thus the Hilbert space $\mathcal{H}$ in which we work in the present paper is given by

$$
\mathcal{H}:=L^{2}\left(\mathbb{R}^{3} ; \mathbb{C}^{4}\right) \otimes \mathcal{K}
$$

We denote by $\mathrm{B}(\mathcal{K})$ the Banach space of all bounded linear operators on $\mathcal{K}$ with domain $\mathcal{K}$. Let $T: \mathbb{R}^{3} \rightarrow \mathrm{B}(\mathcal{K}) ; \mathbb{R}^{3} \ni \boldsymbol{x}=\left(x_{1}, x_{2}, x_{3}\right) \mapsto T(\boldsymbol{x}) \in \mathrm{B}(\mathcal{K})$ be a Borel measurable mapping 
such that, for all $\boldsymbol{x} \in \mathbb{R}^{3}, T(\boldsymbol{x})$ is a non-zero bounded self-adjoint operator on $\mathcal{K}$ such that $\|T\|_{\infty}:=\sup _{\boldsymbol{x} \in \mathbb{R}^{3}}\|T(\boldsymbol{x})\|<\infty$, where $\|T(\boldsymbol{x})\|$ denotes the operator norm of $T(\boldsymbol{x})$.

Example 1. In the original CQSM, $\mathcal{K}=\mathbb{C}^{2}$ and $T(\boldsymbol{x})=\boldsymbol{\tau} \cdot \boldsymbol{n}(\boldsymbol{x})$, where $\boldsymbol{n}: \mathbb{R}^{3} \rightarrow \mathbb{R}^{3}$ is a measurable vector field with $|\boldsymbol{n}(\boldsymbol{x})|=1$, a.e. (almost everywhere) $\boldsymbol{x} \in \mathbb{R}^{3}$ and $\boldsymbol{\tau}=\left(\tau_{1}, \tau_{2}, \tau_{3}\right)$ is the set of the Pauli matrices.

We denote by $\left\{\alpha_{1}, \alpha_{2}, \alpha_{3}, \beta\right\}$ the Dirac matrices, i.e., $4 \times 4$-Hermitian matrices satisfying

$$
\left\{\alpha_{j}, \alpha_{k}\right\}=2 \delta_{j k}, \quad\left\{\alpha_{j}, \beta\right\}=0, \quad \beta^{2}=1, \quad j, k=1,2,3,
$$

where $\{A, B\}:=A B+B A$.

Let $F: \mathbb{R}^{3} \rightarrow \mathbb{R}$ be measurable, a.e., finite and

$$
U_{F}:=(\cos F) \otimes I+i(\sin F) \gamma_{5} \otimes T,
$$

where $I$ denotes identity and $\gamma_{5}:=-i \alpha_{1} \alpha_{2} \alpha_{3}$. We set $\boldsymbol{\alpha}:=\left(\alpha_{1}, \alpha_{2}, \alpha_{3}\right)$ and $\nabla:=\left(D_{1}, D_{2}, D_{3}\right)$ with $D_{j}$ being the generalized partial differential operator in the variable $x_{j}$. Then the one particle Hamiltonian of a generalized CQSM is defined by

$$
H:=-i \boldsymbol{\alpha} \cdot \nabla \otimes I+M(\beta \otimes I) U_{F}
$$

acting in the Hilbert space $\mathcal{H}$. For a linear operator $L$, we denote its domain by $D(L)$. It is well-known that $-i \boldsymbol{\alpha} \cdot \nabla$ is self-adjoint with $D(-i \boldsymbol{\alpha} \cdot \nabla)=\cap_{j=1}^{3} D\left(D_{j}\right)$. Since the operator $M(\beta \otimes I) U_{F}$ is bounded and self-adjoint, it follows that $H$ is self-adjoint with domain $D(H)=$ $\cap_{j=1}^{3} D\left(D_{j} \otimes I\right)=H^{1}\left(\mathbb{R}^{3} ; \mathbb{C}^{4} \otimes \mathcal{K}\right)$, the Sobolev space of order 1 consisting of $\mathbb{C}^{4} \otimes \mathcal{K}$-valued measurable functions on $\mathbb{R}^{3}$. In the context of the CQSM, the function $F$ is called a profile function. In what follows we sometimes omit the symbol of tensor product $\otimes$ in writing equations down.

Example 2. Usually profile functions are assumed to be rotation invariant with boundary conditions

$$
F(0)=-\pi, \quad \lim _{|\boldsymbol{x}| \rightarrow \infty} F(\boldsymbol{x})=0 .
$$

The following are concrete examples [6]:

$$
\begin{array}{ll}
\text { (I) } & F(\boldsymbol{x})=-\pi \exp (-|\boldsymbol{x}| / R), \quad R=0.55 \times 10^{-15} \mathrm{~m} ; \\
\text { (II) } \quad F(\boldsymbol{x})=-\pi\left\{a_{1} \exp \left(-|\boldsymbol{x}| / R_{1}\right)+a_{2} \exp \left(-|\boldsymbol{x}|^{2} / R_{2}^{2}\right)\right\}, \\
& a_{1}=0.65, \quad R_{1}=0.58 \times 10^{-15} \mathrm{~m}, \quad a_{2}=0.35, \quad R_{2}=\sqrt{0.3} \times 10^{-15} \mathrm{~m} ; \\
\text { (III) } & F(\boldsymbol{x})=-\pi\left(1-\frac{|\boldsymbol{x}|}{\sqrt{\lambda^{2}+|\boldsymbol{x}|^{2}}}\right), \quad \lambda=\sqrt{0.4} \times 10^{-15} \mathrm{~m} .
\end{array}
$$

We say that a self-adjoint operator $A$ on $\mathcal{H}$ has chiral symmetry if $\gamma_{5} A \subset A \gamma_{5}$.

Proposition 1. The Hamiltonian $H$ has no chiral symmetry.

Proof. It is easy to check that, for all $\psi \in D(H), \gamma_{5} \psi \in D(H)$ and $\left[\gamma_{5}, H\right] \psi=2 M \gamma_{5} \beta U_{F} \psi$. Note that $U_{F} \neq 0$. Hence, $\left[\gamma_{5}, H\right] \neq 0$ on $D(H)$.

We note that, if $F$ and $T$ are differentiable on $\mathbb{R}^{3}$ with $\sup _{\boldsymbol{x} \in \mathbb{R}^{3}}\left|\partial_{j} F(\boldsymbol{x})\right|<\infty$ and $\sup _{\boldsymbol{x} \in \mathbb{R}^{3}}\left\|\partial_{j} T(\boldsymbol{x})\right\|<\infty$ $(j=1,2,3)$, then the square of $H$ takes the form

$$
H^{2}=\left(-\Delta+M^{2}\right) \otimes I-i M \beta \boldsymbol{\alpha} \cdot\left(\nabla U_{F}\right)+M^{2} \sin ^{2} F \otimes\left(T^{2}-I\right) .
$$

This is a Schrödinger operator with an operator-valued potential. 


\section{Operator matrix representation}

For more detailed analyses of the model, it is convenient to work with a suitable representation of the Dirac matrices. Here we take the following representation of $\alpha_{j}$ and $\beta$ (the Weyl representation):

$$
\alpha_{j}=\left(\begin{array}{cc}
\sigma_{j} & 0 \\
0 & -\sigma_{j}
\end{array}\right), \quad \beta=\left(\begin{array}{cc}
0 & 1 \\
1 & 0
\end{array}\right)
$$

where $\sigma_{1}, \sigma_{2}$ and $\sigma_{3}$ are the Pauli matrices. Let $\boldsymbol{\sigma}:=\left(\sigma_{1}, \sigma_{2}, \sigma_{3}\right)$ and

$$
\Phi_{F}:=(\cos F) \otimes I+i(\sin F) \otimes T .
$$

Then we have the following operator matrix representation for $H$ :

$$
H=\left(\begin{array}{cc}
-i \boldsymbol{\sigma} \cdot \nabla & M \Phi_{F}^{*} \\
M \Phi_{F} & i \boldsymbol{\sigma} \cdot \nabla
\end{array}\right) .
$$

\section{Supersymmetric aspects}

Let $\xi: \mathbb{R}^{3} \rightarrow \mathrm{B}(\mathcal{K})$ be measurable such that, for all $\boldsymbol{x} \in \mathbb{R}^{3}, \xi(\boldsymbol{x})$ is a bounded self-adjoint operator on $\mathcal{K}$ and $\xi(\boldsymbol{x})^{2}=I, \forall \boldsymbol{x} \in \mathbb{R}^{3}$. Let

$$
\Gamma(\boldsymbol{x}):=i \gamma_{5} \beta \otimes \xi(\boldsymbol{x}), \quad \boldsymbol{x} \in \mathbb{R}^{3} .
$$

We define an operator $\hat{\Gamma}$ on $\mathcal{H}$ by

$$
(\hat{\Gamma} \psi)(\boldsymbol{x}):=\Gamma(\boldsymbol{x}) \psi(\boldsymbol{x}), \quad \psi \in \mathcal{H}, \quad \text { a.e. } \boldsymbol{x} \in \mathbb{R}^{3} .
$$

The following fact is easily proven:

Lemma 1. The operator $\hat{\Gamma}$ is self-adjoint and unitary, i.e., it is a grading operator on $\mathcal{H}$ : $\hat{\Gamma}^{*}=\hat{\Gamma}, \hat{\Gamma}^{2}=I$.

Theorem 1. Suppose that $\xi$ is strongly differentiable with $\sup _{\boldsymbol{x} \in \mathbb{R}^{3}}\left\|\partial_{j} \xi(\boldsymbol{x})\right\|<\infty(j=1,2,3)$ and

$$
\sum_{j=1}^{3} \alpha_{j} \otimes D_{j} \xi(\boldsymbol{x})=M \gamma_{5} \beta\{\xi(\boldsymbol{x}), T(\boldsymbol{x})\} \sin F(\boldsymbol{x}) .
$$

Then $\hat{\Gamma} D(H) \subset D(H)$ and $\{\hat{\Gamma}, H\} \psi=0, \forall \psi \in D(H)$.

Proof. For all $\psi \in D_{0}:=C_{0}^{\infty}\left(\mathbb{R}^{3}\right) \otimes_{\text {alg }}\left(\mathbb{C}^{4} \otimes \mathcal{K}\right)\left(\otimes_{\text {alg }}\right.$ denotes algebraic tensor product), we have

$$
D_{j} \hat{\Gamma} \psi=i \gamma_{5} \beta \otimes\left(D_{j} \xi\right) \psi+i \gamma_{5} \beta \otimes \xi\left(D_{j} \psi\right) .
$$

By a limiting argument using the fact that $D_{0}$ is a core of $D_{j} \otimes I$, we can show that, for all $\psi \in D\left(D_{j}\right), \hat{\Gamma} \psi$ is in $D\left(D_{j}\right)$ and (2) holds. Hence, for all $\psi \in D(H), \hat{\Gamma} \psi \in D(H)$ and (2) holds. Thus we have for all $\psi \in D(H)\{\hat{\Gamma}, H\} \psi=C_{1} \psi+C_{2} \psi$ with $C_{1}:=\sum_{j=1}^{3}\left\{\gamma_{5} \beta \otimes \xi, \alpha_{j} D_{j}\right\}$ and $C_{2}:=i M\left\{\gamma_{5} \beta \otimes \xi, \beta U_{F}\right\}$. Using the fact that $\left\{\gamma_{5}, \beta\right\}=0$ and $\left[\gamma_{5}, \alpha_{j}\right]=0(j=1,2,3)$, we obtain $C_{1} \psi=-\gamma_{5} \beta\left(\sum_{j=1}^{3} \alpha_{j} D_{j} \xi\right) \psi$. Similarly direct computations yield $\left(C_{2} \psi\right)(\boldsymbol{x})=-M \sin F(\boldsymbol{x}) \otimes$ $\{\xi(\boldsymbol{x}), T(\boldsymbol{x})\} \psi(x)$. Thus (1) implies $\{\hat{\Gamma}, H\} \psi=0$. 
Theorem 1 means that, under its assumption, $H$ may be interpreted as a generator of a supersymmetry with respect to $\hat{\Gamma}$.

Example 3. Consider the case $\mathcal{K}=\mathbb{C}^{2}$. Let $f, g: \mathbb{R}^{3} \rightarrow \mathbb{R}$ be a continuously differentiable function such that

$$
\left(1+C^{2}\right) f(\boldsymbol{x})^{2}+g(\boldsymbol{x})^{2}=1 .
$$

with a real constant $C \neq 0$ and $\boldsymbol{n}(\boldsymbol{x}):=(f(\boldsymbol{x}), C f(\boldsymbol{x}), g(\boldsymbol{x}))$. Then $|\boldsymbol{n}(\boldsymbol{x})|=1, \forall \boldsymbol{x} \in \mathbb{R}^{3}$. Let

$$
\xi:=\frac{C}{\sqrt{1+C^{2}}} \tau_{1}-\frac{1}{\sqrt{1+C^{2}}} \tau_{2}, \quad T(\boldsymbol{x}):=\boldsymbol{\tau} \cdot \boldsymbol{n}(\boldsymbol{x}) .
$$

Then $\xi^{2}=I$ and $(\xi, T)$ satisfies $(1)$.

To state spectral properties of $H$, we recall some definitions. For a self-adjoint operator $S$, we denote by $\sigma(S)$ the spectrum of $S$. The point spectrum of $S$, i.e., the set of all the eigenvalues of $S$ is denoted $\sigma_{\mathrm{p}}(S)$. An isolated eigenvalue of $S$ with finite multiplicity is called a discrete eigenvalue of $S$. We denote by $\sigma_{\mathrm{d}}(S)$ the set of all the discrete eigenvalues of $S$. The set $\sigma_{\text {ess }}(S):=\sigma(S) \backslash \sigma_{\mathrm{d}}(S)$ is called the essential spectrum of $S$.

Theorem 2. Under the same assumption as in Theorem 1, the following holds:

(i) $\sigma(H)$ is symmetric with respect to the origin of $\mathbb{R}$, i.e., if $\lambda \in \sigma(H)$, then $-\lambda \in \sigma(H)$.

(ii) $\sigma_{\#}(H)(\#=\mathrm{p}, \mathrm{d})$ is symmetric with respect to the origin of $\mathbb{R}$ with

$$
\operatorname{dim} \operatorname{ker}(H-\lambda)=\operatorname{dim} \operatorname{ker}(H-(-\lambda))
$$

for all $\lambda \in \sigma_{\#}(H)$.

(iii) $\sigma_{\mathrm{ess}}(H)$ is symmetric with respect to the origin of $\mathbb{R}$.

Proof. Theorem 1 implies a unitary equivalence of $H$ and $-H\left(\hat{\Gamma} H \hat{\Gamma}^{-1}=-H\right)$. Thus the desired results follow.

Remark 1. Suppose that the assumption of Theorem 1 holds. In view of supersymmetry breaking, it is interesting to compute $\operatorname{dim} \operatorname{ker} H$. This is related to the index problem: Let

$$
\mathcal{H}_{+}:=\operatorname{ker}(\hat{\Gamma}-1), \quad \mathcal{H}_{-}:=\operatorname{ker}(\hat{\Gamma}+1)
$$

and

$$
H_{ \pm}:=H \mid \mathcal{H}_{ \pm}
$$

Then $H_{+}$(resp. $\left.H_{-}\right)$is a densely defined closed linear operator from $\mathcal{H}_{+}$(resp. $\left.\mathcal{H}_{-}\right)$to $\mathcal{H}_{-}$ (resp. $\mathcal{H}_{+}$) with $D\left(H_{+}\right)=D(H) \cap \mathcal{H}_{+}\left(\right.$resp. $D\left(H_{-}\right)=D(H) \cap D\left(H_{-}\right)$). Obviously

ker $H=\operatorname{ker} H_{+} \oplus \operatorname{ker} H_{-}$.

The analytical index of $H_{+}$is defined by

$$
\operatorname{index}\left(H_{+}\right):=\operatorname{dim} \operatorname{ker} H_{+}-\operatorname{dim} \operatorname{ker} H_{+}^{*},
$$

provided that at least one of $\operatorname{dim} \operatorname{ker} H_{+}$and $\operatorname{dim} \operatorname{ker} H_{+}^{*}$ is finite. We conjecture that, for a class of $F$ and $T$, index $\left(H_{+}\right)=0$. 


\section{The essential spectrum and finiteness of the discrete spectrum of $\boldsymbol{H}$}

\subsection{Structure of the spectrum of $H$}

Theorem 3. Suppose that $\operatorname{dim} \mathcal{K}<\infty$ and

$$
\lim _{|\boldsymbol{x}| \rightarrow \infty} F(\boldsymbol{x})=0
$$

Then

$$
\begin{aligned}
& \sigma_{\text {ess }}(H)=(-\infty,-M] \cup[M, \infty), \\
& \sigma_{\mathrm{d}}(H) \subset(-M, M) .
\end{aligned}
$$

Proof. We can rewrite $H$ as $H=H_{0} \otimes I+V$ with $H_{0}:=-i \boldsymbol{\alpha} \cdot \nabla+M \beta$ and $V:=M(\beta \otimes I)$ $\left(U_{F}-I\right)$. We denote by $\chi_{R}(R>0)$ the characteristic function of the set $\left\{\boldsymbol{x} \in \mathbb{R}^{3}|| \boldsymbol{x} \mid<R\right\}$. It is well-known that, for all $z \in \mathbb{C} \backslash \mathbb{R},\left(H_{0}-z\right)^{-1} \chi_{R}$ is compact [7, Lemma 4.6]. Since $\mathcal{K}$ is finite dimensional, it follows that $\left(H_{0} \otimes I-z\right)^{-1} \chi_{R} \otimes I$ is compact. We have

$$
\|V(\boldsymbol{x})\| \leq M\left(|\cos F(\boldsymbol{x})-1|+|\sin F(\boldsymbol{x})|\|T\|_{\infty}\right) \leq M\left(\frac{|F(\boldsymbol{x})|^{2}}{2}+|F(\boldsymbol{x})|\|T\|_{\infty}\right) .
$$

Hence, by (3), we have $\lim _{R \rightarrow \infty} \sup _{|\boldsymbol{x}|>R}\|V(\boldsymbol{x})\|=0$. Then, in the same way as in the method described on [7, pp. 115-117], we can show that, for all $z \in \mathbb{C} \backslash \mathbb{R},(H-z)^{-1}-\left(H_{0} \otimes I-z\right)^{-1}$ is compact. Hence, by a general theorem (e.g., [7, Theorem 4.5]), $\sigma_{\text {ess }}(H)=\sigma_{\text {ess }}\left(H_{0} \otimes I\right)$. Since $\sigma_{\text {ess }}\left(H_{0}\right)=(-\infty,-M] \cup[M, \infty)([7$, Theorem 1.1]), we obtain (4). Relation (5) follows from (4) and $\sigma_{\mathrm{d}}(H)=\sigma(H) \backslash \sigma_{\mathrm{ess}}(H)$.

\subsection{Bound for the number of discrete eigenvalues of $H$}

Suppose that $\operatorname{dim} \mathcal{K}<\infty$ and (3) holds. Then, by Theorem 3, we can define the number of discrete eigenvalues of $H$ counting multiplicities:

$$
N_{H}:=\operatorname{dim} \operatorname{Ran} E_{H}((-M, M)) \text {, }
$$

where $E_{H}$ is the spectral measure of $H$.

To estimate an upper bound for $N_{H}$, we introduce a hypothesis for $F$ and $T$ :

\section{Hypothesis (A).}

(i) $T(\boldsymbol{x})^{2}=I, \forall \boldsymbol{x} \in \mathbb{R}^{3}$ and $T$ is strongly differentiable with $\sum_{j=1}^{3}\left(D_{j} T(\boldsymbol{x})\right)^{2}$ being a multiplication operator by a scalar function on $\mathbb{R}^{3}$.

(ii) $F \in C^{1}\left(\mathbb{R}^{3}\right)$.

(iii) $\sup _{\boldsymbol{x} \in \mathbb{R}^{3}}\left|D_{j} F(\boldsymbol{x})\right|<\infty, \sup _{\boldsymbol{x} \in \mathbb{R}^{3}}\left\|D_{j} T(\boldsymbol{x})\right\|<\infty(j=1,2,3)$.

Under this assumption, we can define

$$
V_{F}(\boldsymbol{x}):=\sqrt{|\nabla F(\boldsymbol{x})|^{2}+\sum_{j=1}^{3}\left(D_{j} T(\boldsymbol{x})\right)^{2} \sin ^{2} F(\boldsymbol{x})} .
$$


Theorem 4. Let $\operatorname{dim} \mathcal{K}<\infty$. Assume (3) and Hypothesis (A). Suppose that

$$
C_{F}:=\int_{\mathbb{R}^{6}} \frac{V_{F}(\boldsymbol{x}) V_{F}(\boldsymbol{y})}{|\boldsymbol{x}-\boldsymbol{y}|^{2}} d \boldsymbol{x} d \boldsymbol{y}<\infty .
$$

Then $N_{H}$ is finite with

$$
N_{H} \leq \frac{(\operatorname{dim} \mathcal{K}) M^{2} C_{F}}{4 \pi^{2}} .
$$

A basic idea for the proof of Theorem 4 is as follows. Let

$$
L(F):=H^{2}-M^{2}
$$

Then we have

$$
L(F)=-\Delta+M\left(\begin{array}{cc}
0 & W_{F}^{*} \\
W_{F} & 0
\end{array}\right)
$$

with $W_{F}:=i \boldsymbol{\sigma} \cdot \nabla \Phi_{F}$. Note that

$$
W_{F}^{*} W_{F}=W_{F} W_{F}^{*}=V_{F}^{2} .
$$

Let

$$
L_{0}(F):=-\Delta-M V_{F} .
$$

For a self-adjoint operator $S$, we introduce a set

$$
N_{-}(S):=\text { the number of negative eigenvalues of } S \text { counting multiplicities. }
$$

The following is a key lemma:

\section{Lemma 2.}

$$
N_{H} \leq N_{-}(L(F)) \leq N_{-}\left(L_{0}(F)\right) .
$$

Proof. For each $\lambda \in \sigma_{\mathrm{d}}(H) \cap(-M, M)$, we have $\operatorname{ker}(H-\lambda) \subset \operatorname{ker}\left(L(F)-E_{\lambda}\right)$ with $E_{\lambda}=$ $\lambda^{2}-M^{2}<0$. Hence the first inequality of (7) follows. The second inequality of (7) can be proven in the same manner as in the proof of [1, Lemma 3.3], which uses the min-max principle.

On the other hand, one has

$$
N_{-}\left(L_{0}(F)\right) \leq \frac{(\operatorname{dim} \mathcal{K}) M^{2} C_{F}}{4 \pi^{2}}
$$

(the Birman-Schwinger bound [4, Theorem XIII.10]). In this way we can prove Theorem 4.

As a direct consequence of Theorem 4, we have the following fact on the absence of discrete eigenvalues of $H$ :

Corollary 1. Assume (3) and Hypothesis (A). Let $(\operatorname{dim} \mathcal{K}) M^{2} C_{F}<4 \pi^{2}$. Then $\sigma_{\mathrm{d}}(H)=\varnothing$, i.e., $H$ has no discrete eigenvalues. 


\section{Existence of discrete ground states}

Let $A$ be a self-adjoint operator on a Hilbert space and bounded from below. Then

$$
E_{0}(A):=\inf \sigma(A)
$$

is finite. We say that $A$ has a ground state if $E_{0}(A) \in \sigma_{\mathrm{p}}(A)$. In this case, a non-zero vector in $\operatorname{ker}\left(A-E_{0}(A)\right)$ is called a ground state of $A$. Also we say that $A$ has a discrete ground state if $E_{0}(A) \in \sigma_{\mathrm{d}}(A)$.

\section{Definition 1. Let}

$$
E_{0}^{+}(H):=\inf [\sigma(H) \cap[0, \infty)], \quad E_{0}^{-}(H):=\sup [\sigma(H) \cap(-\infty, 0]] .
$$

(i) If $E_{0}^{+}(H)$ is an eigenvalue of $H$, then we say that $H$ has a positive energy ground state and we call a non-zero vector in $\operatorname{ker}\left(H-E_{0}^{+}(H)\right)$ a positive energy ground state of $H$.

(ii) If $E_{0}^{-}(H)$ is an eigenvalue of $H$, then we say that $H$ has a negative energy ground state and we call a non-zero vector in $\operatorname{ker}\left(H-E_{0}^{-}(H)\right)$ a negative energy ground state of $H$.

(iii) If $E_{0}^{+}(H)$ (resp. $\left.E_{0}^{-}(H)\right)$ is a discrete eigenvalue of $H$, then we say that $H$ has a discrete positive (resp. negative) energy ground state.

Remark 2. If the spectrum of $H$ is symmetric with respect to the origin of $\mathbb{R}$ as in Theorem 2 , then $E_{0}^{+}(H)=-E_{0}^{-}(H)$, and $H$ has a positive energy ground state if and only if it has a negative energy ground state.

Assume Hypothesis (A). Then the operators

$$
S_{ \pm}(F):=-\Delta \pm M\left(D_{3} \cos F\right)
$$

are self-adjoint with $D\left(S_{ \pm}(F)\right)=D(\Delta)$ and bounded from below.

As for existence of discrete ground states of the Dirac operator $H$, we have the following theorem:

Theorem 5. Let $\operatorname{dim} \mathcal{K}<\infty$. Assume Hypothesis (A) and (3). Suppose that $E_{0}\left(S_{+}(F)\right)<0$ or $E_{0}\left(S_{-}(F)\right)<0$. Then $H$ has a discrete positive energy ground state or a discrete negative ground state.

Proof. We describe only an outline of proof. We have

$$
\sigma_{\mathrm{ess}}(L(F))=[0, \infty), \quad \sigma_{\mathrm{d}}(L(F)) \subset\left[-M^{2}, 0\right) .
$$

Hence, if $L(F)$ has a discrete eigenvalue, then $H$ has a discrete eigenvalue in $(-M, M)$. By the min-max principle, we need to find a unit vector $\Psi$ such that $\langle\Psi, L(F) \Psi\rangle<0$. Indeed, for each $f \in D(\Delta)$, we can find vectors $\Psi_{f}^{ \pm} \in D(L(F))$, such that $\left\langle\Psi_{f}^{ \pm}, L(F) \Psi_{f}^{ \pm}\right\rangle=\left\langle f, S_{ \pm} f\right\rangle$. By the present assumption, there exists a non-zero vector $f_{0} \in D(\Delta)$ such that $\left\langle f_{0}, S_{+}(F) f_{0}\right\rangle<0$ or $\left\langle f_{0}, S_{-}(F) f_{0}\right\rangle<0$. Thus the desired results follow.

To find a class of $F$ such that $E_{0}\left(S_{+}(F)\right)<0$ or $E_{0}\left(S_{-}(F)\right)<0$, we proceed as follows. For a constant $\varepsilon>0$ and a function $f$ on $\mathbb{R}^{d}$, we define a function $f_{\varepsilon}$ on $\mathbb{R}^{d}$ by

$$
f_{\varepsilon}(x):=f(\varepsilon x), \quad x \in \mathbb{R}^{d} .
$$

The following are key Lemmas. 
Lemma 3. Let $V: \mathbb{R}^{d} \rightarrow \mathbb{R}$ be in $L_{\mathrm{loc}}^{2}\left(\mathbb{R}^{d}\right)$ and

$$
S_{\varepsilon}:=-\Delta+V_{\varepsilon}
$$

Suppose that:

(i) For all $\varepsilon>0, S_{\varepsilon}$ is self-adjoint, bounded below and $\sigma_{\mathrm{ess}}\left(S_{\varepsilon}\right) \subset[0, \infty)$.

(ii) There exists a nonempty open set $\Omega \subset\left\{x \in \mathbb{R}^{d} \mid V(x)<0\right\}$.

Then then there exists a constant $\varepsilon_{0}>0$ such that, for all $\varepsilon \in\left(0, \varepsilon_{0}\right), S_{\varepsilon}$ has a discrete ground state.

Proof. A basic idea for the proof of this lemma is to use the min-max principle (see [1, Lemma 4.3]).

Lemma 4. $V: \mathbb{R}^{d} \rightarrow \mathbb{R}$ be continuous with $V(x) \rightarrow 0(|x| \rightarrow \infty)$. Suppose that $\left\{x \in \mathbb{R}^{d} \mid V(x)<\right.$ $0\} \neq \varnothing$. Then:

(i) $-\Delta+V$ is self-adjoint and bounded below.

(ii) $\sigma_{\text {ess }}(-\Delta+V)=[0, \infty)$.

(iii) $S_{\varepsilon}$ has a discrete ground state for all $\varepsilon \in\left(0, \varepsilon_{0}\right)$ with some $\varepsilon_{0}>0$.

Proof. The facts (i) and (ii) follow from the standard theory of Schrödinger operators. Part (iii) follow from a simple application of Lemma 3 (for more details, see the proof of [1, Lemma 4.4]).

We now consider a one-parameter family of Dirac operators:

$$
H_{\varepsilon}:=(-i) \boldsymbol{\alpha} \cdot \nabla+\frac{1}{\varepsilon} M(\beta \otimes I) U_{F_{\varepsilon}} .
$$

Theorem 6. Let $\operatorname{dim} \mathcal{K}<\infty$. Assume Hypothesis (A) and (3). Suppose that $D_{3} \cos F$ is not identically zero. Then there exists a constant $\varepsilon_{0}>0$ such that, for all $\varepsilon \in\left(0, \varepsilon_{0}\right)$, $H_{\varepsilon}$ has a discrete positive energy ground state or a discrete negative ground state.

Proof. This follows from Theorem 5 and Lemma 4 (for more details, see the proof of [1, Theorem 4.5]).

\section{Symmetry reduction of $H$}

Let $T_{1}, T_{2}$ and $T_{3}$ be bounded self-adjoint operators on $\mathcal{K}$ satisfying

$$
\begin{aligned}
& T_{j}^{2}=I, \quad j=1,2,3, \\
& T_{1} T_{2}=i T_{3}, \quad T_{2} T_{3}=i T_{1}, \quad T_{3} T_{1}=i T_{2} .
\end{aligned}
$$

Then it is easy to see that the anticommutation relations

$$
\left\{T_{j}, T_{k}\right\}=2 \delta_{j k} I, \quad j, k=1,2,3
$$

hold. Since each $T_{j}$ is a unitary self-adjoint operator with $T_{j} \neq \pm I$, it follows that

$$
\sigma\left(T_{j}\right)=\sigma_{\mathrm{p}}\left(T_{j}\right)=\{ \pm 1\} .
$$

We set $\boldsymbol{T}=\left(T_{1}, T_{2}, T_{3}\right)$. 
In this section we consider the case where $T(\boldsymbol{x})$ is of the following form:

$$
T(\boldsymbol{x})=\boldsymbol{n}(\boldsymbol{x}) \cdot \boldsymbol{T},
$$

where $\boldsymbol{n}(\boldsymbol{x})$ is the vector field in Example 1. We use the cylindrical coordinates for points $\boldsymbol{x}=\left(x_{1}, x_{2}, x_{3}\right) \in \mathbb{R}^{3}$ :

$$
x_{1}=r \cos \theta, \quad x_{2}=r \sin \theta, \quad x_{3}=z,
$$

where $\theta \in[0,2 \pi), r>0$. We assume the following:

Hypothesis (B). There exists a continuously differentiable function $G:(0, \infty) \times \mathbb{R} \rightarrow \mathbb{R}$ such that

(i) $F(\boldsymbol{x})=G(r, z), \boldsymbol{x} \in \mathbb{R}^{3} \backslash\{0\}$;

(ii) $\lim _{r+|z| \rightarrow \infty} G(r, z)=0$;

(iii) $\sup _{r>0, z \in \mathbb{R}}(|\partial G(r, z) / \partial r|+|\partial G(r, z) / \partial z|)<\infty$.

We take the vector field $n: \mathbb{R}^{3} \rightarrow \mathbb{R}^{3}$ to be of the form

$$
\boldsymbol{n}(\boldsymbol{x}):=(\sin \Theta(r, z) \cos (m \theta), \sin \Theta(r, z) \sin (m \theta), \cos \Theta(r, z)),
$$

where $\Theta:(0, \infty) \times \mathbb{R} \rightarrow \mathbb{R}$ is continuous and $m$ is a natural number.

Let $L_{3}$ be the third component of the angular momentum acting in $L^{2}\left(\mathbb{R}^{3}\right)$ and

$$
K_{3}:=L_{3} \otimes I+\frac{1}{2} \Sigma_{3} \otimes I+\frac{m}{2} I \otimes T_{3}
$$

with $\Sigma_{3}:=\sigma_{3} \oplus \sigma_{3}$. It is easy to see that $K_{3}$ is a self-adjoint operator acting in $\mathcal{H}$.

Lemma 5. Assume that

$$
\Theta(\varepsilon r, \varepsilon z)=\Theta(r, z), \quad(r, z) \in(0, \infty) \times \mathbb{R}, \quad \varepsilon>0 .
$$

Then, for all $t \in \mathbb{R}$ and $\varepsilon>0$, the operator equality

$$
e^{i t K_{3}} H_{\varepsilon} e^{-i t K_{3}}=H_{\varepsilon}
$$

holds.

Proof. Similar to the proof of [1, Lemma 5.2]. We remark that, in the calculation of

$$
e^{i t K_{3}} T(\boldsymbol{x}) e^{-i t K_{3}}=\sum_{j=1}^{3} e^{i t L_{3}} n_{j}(\boldsymbol{x}) e^{-i t L_{3}} e^{i t m T_{3}} T_{j} e^{-i t m T_{3}},
$$

the following formulas are used:

$$
\left(T_{1} \cos m t-T_{2} \sin m t\right) e^{i t m T_{3}}=T_{1}, \quad\left(T_{1} \sin m t+T_{2} \cos m t\right) e^{i t m T_{3}}=T_{2} .
$$

Definition 2. We say that two self-adjoint operators on a Hilbert space strongly commute if their spectral measures commute.

Lemma 6. Assume (9). Then, for all $\varepsilon>0, H_{\varepsilon}$ and $K_{3}$ strongly commute.

Proof. By (10) and the functional calculus, we have for all $s, t \in \mathbb{R} e^{i t K_{3}} e^{i s H_{\varepsilon}} e^{-i t K_{3}}=e^{i s H_{\varepsilon}}$, which is equivalent to $e^{i t K_{3}} e^{i s H_{\varepsilon}}=e^{i s H_{\varepsilon}} e^{i t K_{3}}, s, t \in \mathbb{R}$. By a general theorem (e.g., [3, Theorem VIII.13]), this implies the strong commutativity of $K_{3}$ and $H_{\varepsilon}$. 
Lemma 6 implies that $H_{\varepsilon}$ is reduced by eigenspaces of $K_{3}$. Note that

$$
\sigma\left(K_{3}\right)=\sigma_{\mathrm{p}}\left(K_{3}\right)=\left\{\ell+\frac{s}{2}+\frac{m t}{2} \mid \ell \in \mathbb{Z}, s= \pm 1, t= \pm 1\right\} .
$$

The eigenspace of $K_{3}$ with eigenvalue $\ell+(s / 2)+(m t / 2)$ is given by

$$
\mathcal{M}_{\ell, s, t}:=\mathcal{M}_{\ell} \otimes \mathcal{C}_{s} \otimes \mathcal{T}_{t}
$$

with $\mathcal{C}_{s}:=\operatorname{ker}\left(\Sigma_{3}-s\right)$ and $\mathcal{T}_{t}:=\operatorname{ker}\left(T_{3}-t\right)$. Then $\mathcal{H}$ has the orthogonal decomposition

$$
\mathcal{H}=\oplus_{\ell \in \mathbb{Z}, s, t \in\{ \pm 1\}} \mathcal{M}_{\ell, s, t} .
$$

Thus we have:

Lemma 7. Assume (9). Then, for all $\varepsilon>0, H_{\varepsilon}$ is reduced by each $\mathcal{M}_{\ell, s, t}$.

We denote by $H_{\varepsilon}(\ell, s, t)$ by the reduced part of $H_{\varepsilon}$ to $\mathcal{M}_{\ell, s, t}$ and set

$$
H(\ell, s, t):=H_{1}(\ell, s, t) \text {. }
$$

For $s= \pm 1$ and $\ell \in \mathbb{Z}$, we define

$$
L_{s}(G, \ell):=-\frac{\partial^{2}}{\partial r^{2}}-\frac{1}{r} \frac{\partial}{\partial r}+\frac{\ell^{2}}{r^{2}}+\frac{\partial^{2}}{\partial z^{2}}+s M D_{z} \cos G
$$

acting in $L^{2}((0, \infty) \times \mathbb{R}, r d r d z)$ with domain

$$
D\left(L_{s}(G, \ell)\right):=C_{0}^{\infty}((0, \infty) \times \mathbb{R})
$$

and set

$$
\mathcal{E}_{0}\left(L_{s}(G, \ell)\right):=\inf _{f \in C_{0}^{\infty}((0, \infty) \times \mathbb{R}),\|f\|_{L^{2}((0, \infty) \times \mathbb{R}, r d r d z)}=1}\left\langle f, L_{s}(G, \ell) f\right\rangle .
$$

The following theorem is concerned with the existence of discrete ground states of $H(\ell, s, t)$.

Theorem 7. Assume Hypothesis (B) and (9). Fix an $\ell \in \mathbb{Z}$ arbitrarily, $s= \pm 1$ and $t= \pm 1$. Suppose that $\operatorname{dim} \mathcal{T}_{t}<\infty$ and

$$
\mathcal{E}_{0}\left(L_{s}(G, \ell)\right)<0 \text {. }
$$

Then $H(\ell, s, t)$ has a discrete positive energy ground state or a discrete negative ground state.

Proof. Similar to the proof of Theorem 5 (for more details, see the proof of [1, Theorem 5.5]).

Theorem 8. Assume Hypothesis (B) and (9). Suppose that $\operatorname{dim} \mathcal{T}_{t}<\infty$ and that $D_{z} \cos G$ is not identically zero. Then, for each $\ell \in \mathbb{Z}$, there exists a constant $\varepsilon_{\ell}>0$ such that, for all $\varepsilon \in\left(0, \varepsilon_{\ell}\right)$, each $H_{\varepsilon}(\ell, s, t)$ has a discrete positive energy ground state or a discrete negative ground state.

Proof. Similar to the proof of Theorem 6 (for more details, see the proof of $[1$, Theorem 5.6]).

Theorem 8 immediately yields the following result:

Corollary 2. Assume Hypothesis (B) and (9). Suppose that $\operatorname{dim} \mathcal{T}_{t}<\infty$ and that $D_{z} \cos G$ is not identically zero. Let $\varepsilon_{\ell}$ be as in Theorem 8 and, for each $n \in \mathbb{N}$ and $k>n(k, n \in \mathbb{Z})$, $\nu_{k, n}:=\min _{n+1 \leq \ell \leq k} \varepsilon_{\ell}$. Then, for each $\varepsilon \in\left(0, \nu_{k, n}\right), H_{\varepsilon}$ has at least $(k-n)$ discrete eigenvalues counting multiplicities.

Proof. Note that $\sigma_{\mathrm{p}}\left(H_{\varepsilon}\right)=\cup_{\ell \in \mathbb{Z}, s, t= \pm 1} \sigma_{\mathrm{p}}\left(H_{\varepsilon}(\ell, s, t)\right)$. 


\section{A unitary transformation}

We go back again to the generalized CQSM defined in Section 2. It is easy to see that the operator

$$
X_{F}:=\frac{1+\gamma_{5}}{2} \exp \left(i F \otimes \frac{T}{2}\right)+\frac{1-\gamma_{5}}{2} \exp \left(-i F \otimes \frac{T}{2}\right)
$$

is unitary. Under Hypothesis (A), we can define the following operator-valued functions:

$$
B_{j}(\boldsymbol{x}):=\frac{1}{2} D_{j}[F(\boldsymbol{x}) T(\boldsymbol{x})], \quad \boldsymbol{x} \in \mathbb{R}^{3}, \quad j=1,2,3 .
$$

We set

$$
\boldsymbol{B}:=\left(B_{1}, B_{2}, B_{3}\right)
$$

and introduce

$$
H(\boldsymbol{B}):=(-i) \boldsymbol{\alpha} \cdot \nabla+M \beta-\boldsymbol{\sigma} \cdot \boldsymbol{B}
$$

acting in $\mathcal{H}$. Since $\boldsymbol{\sigma} \cdot \boldsymbol{B}$ is a bounded self-adjoint operator, $H(\boldsymbol{B})$ is self-adjoint with $D(H(\boldsymbol{B}))=$ $\cap_{j=1}^{3} D\left(D_{j} \otimes I\right)$.

Proposition 2. Assume Hypothesis (A) and that $T(\boldsymbol{x})$ is independent of $\boldsymbol{x}$. Then

$$
X_{F} H X_{F}^{-1}=H(\boldsymbol{B}) \text {. }
$$

Proof. Similar to the proof of [1, Proposition 6.1].

Using this proposition, we can prove the following theorem:

Theorem 9. Let $\operatorname{dim} \mathcal{K}<\infty$. Assume Hypothesis (A) and that $T(\boldsymbol{x})$ is independent of $\boldsymbol{x}$. Suppose that

$$
\lim _{|\boldsymbol{x}| \rightarrow \infty}|\nabla F(\boldsymbol{x})|=0
$$

Then

$$
\sigma_{\mathrm{ess}}(H)=(-\infty,-M] \cup[M, \infty) .
$$

Proof. By Proposition 2, we have $\sigma_{\mathrm{ess}}(H)=\sigma_{\mathrm{ess}}(H(\boldsymbol{B}))$. By the present assumption, $B_{j}(\boldsymbol{x})=$ $D_{j} F(\boldsymbol{x}) T(\mathbf{0}) / 2$. Hence

$$
\sup _{|\boldsymbol{x}|>R}\|\boldsymbol{\sigma} \cdot \boldsymbol{B}(\boldsymbol{x})\| \leq \sum_{j=1}^{3}(\|T(\mathbf{0})\| / 2) \sup _{|\boldsymbol{x}|>R}\left|D_{j} F(\boldsymbol{x})\right| \rightarrow 0 \quad(R \rightarrow \infty) .
$$

Therefore, as in the proof of Theorem 3, we conclude that $\sigma_{\text {ess }}(H(B))=(-\infty,-M] \cup[M, \infty]$. Thus (11) follows. 


\section{Acknowledgements}

The author would like to thank N. Sawado for kindly informing on typical examples of profile functions and comments. This work was supported by the Grant-In-Aid 17340032 for Scientific Research from the JSPS.

[1] Arai A., Hayashi K., Sasaki I., Spectral properties of a Dirac operator in the chiral quark soliton model, J. Math. Phys., 2005, V.46, N 5, 052360, 12 pages.

[2] Kalf H., Yamada O., Essential self-adjointness of $n$-dimensional Dirac operators with a variable mass term, J. Math. Phys., 2001, V.42, 2667-2676.

[3] Reed M., Simon B., Methods of modern mathematical physics I: Functional analysis, New York, Academic Press, 1972.

[4] Reed M., Simon B., Methods of modern mathematical physics IV: Analysis of operators, New York, Academic Press, 1978.

[5] Sawado N., The $S U(3)$ dibaryons in the chiral quark soliton model, Phys. Lett. B, 2002, V.524, $289-296$.

[6] Sawado N., Private communication.

[7] Thaller B., The Dirac equation, Springer-Verlag, 1992. 